\title{
On Sensing-Access Tradeoff in Cognitive Radio Networks
}

\author{
Alexander W. Min and Kang G. Shin \\ Real-Time Computing Laboratory, Dept. of EECS \\ The University of Michigan, Ann Arbor, MI 48109-2121 \\ \{alexmin,kgshin\}@eecs.umich.edu
}

\begin{abstract}
In cognitive radio networks (CRNs), the design of an optimal spectrum sensing scheme is an important problem that has recently been drawing consideration attention. Various sensing-related performance tradeoffs have been studied as an efficient means to maximize the secondary network performance. Despite its importance, however, the sensing-access tradeoffbetween sensing overhead and the MAC-layer contention among secondary users in accessing the thus-discovered spectrum opportunities-has not yet been accounted for. In this paper, we show that the secondary network throughput can be improved significantly by incorporating the sensing-access tradeoff in the design of spectrum sensing. We first introduce a new concept of $(\alpha, \beta)$-contention spectrum sharing and analyze the sensing requirement to meet a certain channel contention constraint by using the improper list-coloring in graph theory. Specifically, we derive the relationship among the sensing requirements, the secondary network density, and the transmission power of secondary users. To maximize the network throughput, we propose a distributed spectrum-sharing algorithm, called Smart Share, which exploits channel contention and heterogeneous channel conditions to maximize the secondary network throughput. We also describe how to realize Smart Share in an 802.11 MAC protocol for its practical use and evaluation. Our simulationbased evaluation shows that, sensing an optimal number of channels for given network density can improve the achievable throughput of Smart Share by up to $60 \%$ over a single-channel sensing strategy.
\end{abstract}

\section{INTRODUCTION}

Cognitive radios (CRs) are key for opportunistic (or dynamic) access to licensed spectrum bands, thus alleviating the spectrum-scarcity problem that is expected to occur in the very near future. This new paradigm of opportunistic spectrum access has now become a reality. In November 2008, for example, the FCC ruled that the DTV white spaces can be used by secondary (unlicensed) devices [1]. Moreover, the IEEE 802.22 WRANs, the first CR-based international air-interface, is also in its final stage of standardization [2]. Realization of CR technology requires resolution of several challenges, of which various aspects of spectrum sensing have been studied extensively, such as cooperative sensing [3], [4], sensing scheduling [5], sensor mobility [6], sensing-parameter optimization [7], [8], security [9], [10], to name a few.

Recently, the design of an "optimal" spectrum-sensing strategy has also received considerable attention. The common design objective of optimal sensing is to maximize the network throughput by minimizing the sensing overhead (e.g., in terms of time and energy) under certain performance constraints, e.g., detection accuracy/delay and interference to the primary users. To this end, various sensing-related performance tradeoffs have been exploited in many different contexts, such as sensor selection [5], [11], sensing scheduling [8], [12], [13], and channel sensing ordering [14]. For example, Liang et al. [7] optimized the sensing time by making the tradeoff between the sensing accuracy and the sensing time overhead in order to maximize the secondary network throughput. These approaches are shown to improve the network throughput significantly by exploiting various tradeoffs with regard to the design or determination of sensing parameters.

Despite its importance, however, the impact of sensing on the secondary users' channel access has received far less attention. Intuitively, while sensing a larger number of channels will allow secondary users to access the medium for a longer period of time with reduced channel contention, it will also increase the sensing time overhead, and vice versa. However, most current sensing schemes do not consider this tradeoff. Recently, Zhao et al. [15] jointly considered sensing and access using partially-observed Markov decision process (POMDP). However, they did not consider the impacts of sensing overhead and channel contention in maximizing the secondary network throughput.

In this paper, we propose contention-aware spectrumsensing at the MAC-layer by making the tradeoff between the sensing overhead and the channel-access time to maximize the secondary network's throughput. For this, we must answer the following two questions:

1) How many available channels should each secondary user discover via spectrum-sensing?

2) How efficiently and fairly should the thus-discovered spectrum opportunities be shared among secondary users?

Answers to these questions will provide us useful insights on the impact of secondary system parameters, such as network density, on the design of spectrum-sensing schemes.

We formulate these as a mixed-integer nonlinear programming (MINLP) problem, which turns out to be NP-hard, so we do not expect to find an optimal solution. Instead, we first capture the sensing-access tradeoff by reassessing the throughput maximization problem with the improper list-coloring in graph theory. To gauge the impact of a secondary network's density on spectrum-sensing, we introduce a new concept of $(\alpha, \beta)$ contention spectrum sharing, under which at least $\beta$ fraction of secondary links have less than $\alpha$ interfering links using the same channel. We then propose a distributed spectrumsharing algorithm, called Smart Share, that maximizes the secondary network's throughput by intelligently sharing the available channels among nearby secondary links. To the best of our knowledge, this is the first to use the improper listcoloring for spectrum sensing and sharing in CRNs. 


\section{A. Contributions}

The main contributions of this paper are summarized as follows.

- Introduction of a new way of designing a spectrumsensing scheme by making the tradeoff between the spectrum sensing and channel access of secondary users. Despite its practical importance, the impact of spectrum sensing on channel contention among secondary users has not been considered adequately before. This new approach provides a useful insight on the design of sensing schemes, improving the performance of secondary networks (see Section IV).

- Analysis of $(\alpha, \beta)$-contention spectrum sharing using the improper list-coloring in graph theory. While the conventional graph coloring is suitable only for interference-free spectrum sharing, the improper list-coloring can capture the tradeoff between sensing overhead and channel access. In particular, we prove that $c=O(\lambda)$ where $c$ and $\lambda$ are the minimum required number of available channels and network density, respectively. ${ }^{1}$ This result indicates that the sensing strategy should adapt to the secondary network density to maximize the network throughput. We also prove that $c=O\left(P_{o}^{2 / \gamma}\right)$ where $P_{o}$ and $\gamma$ are the transmit power of a secondary user and path-loss exponent, respectively. Our analysis results suggest that secondary network parameters, such as network density and transmission power, can greatly affect the sensing efficiency, while they have not been considered before (see Section V).

- Development of a contention-aware distributed spectrumsharing algorithm, called Smartshare, for intelligent sharing of spectrum opportunities. We also describe how to realize Smartshare in the 802.11 MAC protocol, and use it for performance evaluation. Our simulation results show that the performance of Smartshare can be improved significantly by employing an optimal sensing strategy in relation with network density. The results also indicate that SmartShare can further improve the secondary network throughput by exploiting heterogeneous channel conditions due to shadow fading, while incurring negligible communication overhead (see Section VII).

\section{B. Organization}

The remainder of this paper is organized as follows. Section II summarizes the related work and highlights the differences between existing work and our approach. Section III describes the system models and the assumptions used in this paper. Section IV formulates the contention-aware channel sensing and sharing problem as a mixed-integer nonlinear programming problem, and introduces the improper list-coloring problem as a solution approach. A graph-theoretic analysis on the sensing-access tradeoff is presented in Section V. Section VI presents four spectrum-sharing algorithms, including SmartShare, and describes the 802.11-based MAC protocols for their practical use and performance evaluation.

\footnotetext{
${ }^{1}$ In this paper, we use Knuth's asymptotic notation: $f(n)=O(g(n))$ implies the existence of some constant $C$ and integer $N$ such that $f(n) \leq$ $C g(n)$ for $n \geq N$.
}

Section VII analyzes the communication overheads of proposed algorithms, and evaluates their performance for various network environments. Section VIII concludes the paper.

\section{RELATED WORK}

The problem of designing an optimal spectrum-sensing strategy has been studied extensively from a single secondary link's perspective [7], [12], [13]. Liang et al. [7] studied the tradeoff in determining the sensing time, i.e., a longer sensing time provides more accurate sensing results, thus better protection of the primary system, at the cost of reduced throughput. Chang and Liu [12] developed an optimal probing strategy by considering both channel probing cost and channel selection for data transmission. Shu et al. [13] formulated the spectrum-sensing problem as an optimal stopping-time problem to maximize the network throughput. However, none of these considered the need for synchronized sensing (quiet) periods, and thus, they all suffer inaccurate sensing (a.k.a. the sensing exposed terminal problem in [16]). Moreover, their design did not consider the contention among the secondary users sharing available spectrum opportunities.

Recently, the problem of joint spectrum sensing and sharing start to draw the researchers' attention [15], [16], [17]. Zhao et al. [15] proposed a distributed POMDP (partially-observed Markov decision process) framework for joint spectrum sensing and sharing in ad hoc CRNs. However, they did not account for the sensing overhead in throughput performance, nor did they consider the channel contention but assumed that only a single pair of secondary users can access a channel within a time slot. Jia et al. [16] proposed a hardware-constrained MAC (HC-MAC) for ad hoc CRNs acknowledging the need for synchronized sensing periods to achieve sensing accuracy. However, their scheme requires only one-hop neighbors to remain silent during spectrum sensing, yielding inaccurate sensing results due to interference from other concurrentlytransmitting secondary users in the same channel.

Our work is different from the previous work: it aims to design a network-wise sensing strategy that incorporates various practical aspects, such as channel contention, network density, and heterogeneous channel conditions.

\section{System MOdEL AND Assumptions}

This section describes the network, interference, and signal propagation models as well as the assumptions that will be used throughout the paper.

\section{A. Network and Interference Models}

We consider an ad-hoc CRN where primary and secondary users coexist in the same geographical area. We assume that each secondary user is equipped with a single radio interface and can access only one channel at a time. We assume that there is a set $\mathcal{K}=\{1, \ldots, K\}$ of orthogonal, noninterfering licensed channels, e.g., non-adjacent TV channels in VHF/UHF bands. These licensed channels can be opportunistically used by secondary users based on their spatial and temporal availability identified via periodic sensing.

We assume the (binary) protocol interference model for the clarity of presentation. That is, nodes $i$ and $j$ interfere with each other if they are located within the interference range $r_{I}$, i.e., $d_{i j} \leq r_{I}$. We consider the 802.11-like bidirectional interference model where two links $l_{1}$ and $l_{2}$ interfere with 
each other if any of the four sender-receiver pairs are located within the interference range. Then, the impact of secondary interferences can be captured by a conflict graph (a.k.a. interference graph) $G_{c}=\left(V_{c}, E_{c}\right)$ [18] where each vertex of $G_{c}$ is associated with a secondary link, and the set of edges $E_{c}$ represents the interference relations between the secondary links, i.e., $E_{c}=\left\{\left(l_{a b}, l_{c d}\right) \mid l_{a b} \otimes l_{c d}=1 l_{a b}, l_{c d} \in V_{c}\right\}$, where the operator $\otimes$ represents the interference relation between the secondary links, i.e., $l_{1} \otimes l_{2}=1(0)$, if links $l_{1}$ and $l_{2}$ (do not) interfere with each other. We assume that the distribution of active secondary links, i.e., a set of vertices $V_{c}$, in the deployment area $A$ follows a point Poisson distribution with density $\lambda$, i.e., $n_{A} \sim \operatorname{Poi}(k ; \lambda|A|)$. The impact of network density on the sensing strategy will be detailed in Section V.

\section{B. Signal Propagation Model}

The received signal strength at the receiver of secondary link $i$ can be expressed as the following signal propagation model:

$$
P_{i}=P_{o}\left(\frac{d_{o}}{d_{i}}\right)^{\gamma} 10^{\frac{X_{i}}{10}} \quad \text { (Watt), }
$$

where $P_{o}$ is the signal strength at the transmitter of secondary link $i, \gamma$ the path-loss exponent, $d_{o}$ the short reference distance (e.g., $1 \mathrm{~m}$ ), and $d_{i}$ the transmitter-receiver separation. The shadow-fading gain is accounted for in $e^{X_{i}}$ where $X_{i} \sim \mathcal{N}\left(0, \sigma^{2}\right) \forall i$. The log-normal shadow fading is often characterized by its $\mathrm{dB}$-spread, $\sigma_{d B}$, which has the relationship $\sigma=0.1 \log _{10}\left(\sigma_{d B}\right)$. We assume that the channel bandwidth is much larger than the coherent bandwidth, so the effect of multi-path fading can be ignored [19].

\section{Problem Formulation}

In this section, we first describe a time-slotted opportunistic spectrum-access model, and express the individual secondary link throughput. We then formulate the secondary network throughput maximization as MINLP and propose $(\alpha, \beta)$ contention to capture the sensing-access tradeoff.

\section{A. Time-Slotted Opportunistic Spectrum Access}

We assume a time-slotted model with constant time-slot duration $(\tau)$ for opportunistic spectrum access (a.k.a. constant access time (CAT) [12]), which has been widely used in the literature (e.g., [7], [15], [16], [20], [21]). The IEEE 802.22 standard draft [22] also employs such a time-synchronized spectrum-sensing model. Note that this time-slotted opportunistic spectrum-access model is suitable to meet the strict timing requirements for incumbent detection, e.g., 2-second channel detection time (CDT) in 802.22 [23]. Each time slot consists of three phases: (i) channel sensing/probing $\left(T_{P}\right)$, (ii) channel selection $\left(T_{A}\right)$, and (iii) data transmission $\left(T_{D}\right)$, as depicted in Fig. 1. To achieve accurate sensing results, we assume that all the secondary users are synchronized and remain silent during the sensing period. We assume that the shadowing gain is constant for a time slot $\tau$. A key challenge is to make the best tradeoff between the channel-probing overhead $T_{P}$ and the channel access time, i.e., the more available channels, the less contention in channel access during data transmission period $T_{D}$. This tradeoff can be captured by the improper/defective list-coloring problem [24] in graph theory, which will be utilized in Section V.

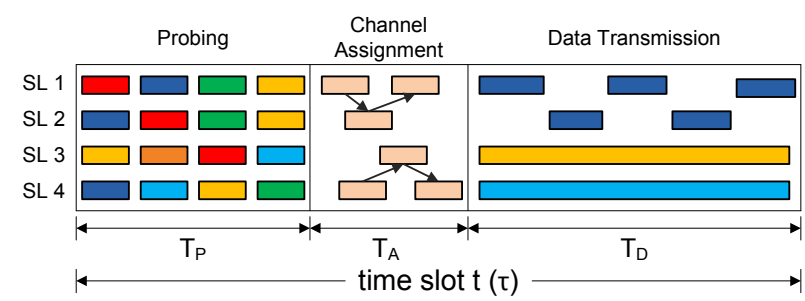

Fig. 1. A time-slotted opportunistic spectrum-access model. The sensing/probing period $T_{P}$ must be synchronized over the entire network to obtain accurate sensing results. Secondary links share the set of available channels discovered via sensing in $T_{A}$ and access the channel via contention in the data transmission period $T_{D}$.

\section{B. Individual Link Throughput under Contention}

Before formulating the network throughput maximization problem, we need to understand the achievable throughput of individual secondary link. For this, we first define the notion of airtime-share in order to incorporate the impact of channel access contention among secondary links.

Definition 1. (AIRTIME SHARE) An airtime share of $a$ secondary link is defined as the expected fraction of time within a time slot the secondary nodes on the link can access the channel.

Given a conflict graph $G_{c}=\left(V_{c}, E_{c}\right)$, let $\phi_{n}(\Lambda)$ denote the achievable airtime share of a secondary link $n \in V_{c}$ under the spectrum-sharing (i.e., channel-assignment) policy $\Lambda: V_{c} \rightarrow$ $\mathcal{K}$. Then, the airtime share of the link can be approximated as:

$$
\phi_{n}(\Lambda) \approx \frac{T_{D}}{\tau} \cdot \frac{1-\delta}{M_{n}(\Lambda)+1},
$$

where $\delta(0<\delta<1)$ denotes channel contention overhead ${ }^{2}$ and $M_{n}(\Lambda)$ denotes the number of interfering secondary links that have been assigned the same channel, i.e.,

$$
M_{n}(\Lambda)=\left|\left\{m \mid \Lambda(m)=\Lambda(n) \quad e_{m n} \in E_{c}\right\}\right| .
$$

In other words, $M_{n}(\Lambda)$ is the number of vertices adjacent to vertex $n$ in the conflict graph.

Then, based on Eqs. (2) and (3), the expected achievable throughput of secondary link $n$ under the spectrum-sharing policy $\Lambda$ can be expressed as:

$$
Z_{n}(\Lambda)=r_{n}(\Lambda) \cdot \phi_{n}(\Lambda)
$$

where $r_{n}(\Lambda)$ is the achievable data rate, which depends on the instantaneous channel condition (e.g., shadow fading).

\section{Utility Maximization Problem}

Based on the above observations, we want to find the optimal spectrum-sharing policy $\Lambda^{*}$ that maximizes the secondary network utility, i.e.,

$$
\Lambda^{*}=\arg \max _{\Lambda}\left[\sum_{n \in V_{c}} \mathcal{U}\left(Z_{n}(\Lambda)\right)\right],
$$

where a different utility function $\mathcal{U}(\cdot)$ can be used to achieve a different level of fairness [25].

\footnotetext{
${ }^{2}$ We assume 802.11-like MAC protocol for channel contention among the secondary nodes assigned the same channel.
} 
Let $\psi_{n, k}$ denote a binary random variable indicating if channel $k \in \mathcal{K}$ is assigned to secondary link $n \in V_{c}$, i.e.,

$$
\psi_{n, k}= \begin{cases}1 & \text { if channel } \mathrm{k} \text { is assigned to secondary link } \mathrm{n} \\ 0 & \text { otheriwse. }\end{cases}
$$

Under the assumptions that the channel probing $\left(T_{P}\right)$ and assignment overheads $\left(T_{A}\right)$ are constant, given the number of probing channels and employed algorithm, the problem of maximizing total network throughput, i.e., $\mathcal{U}(x)=x$, can be formulated as the following optimization problem (P1):

$$
\begin{array}{ll}
\text { Maximize } & \sum_{n \in V_{c}} \sum_{k \in C_{n}} r_{n, k} \cdot \phi_{n, k} \cdot \psi_{n, k}, \\
\text { subject to } & r_{n, k}=B \cdot \log \left(1+\frac{P_{n, k}}{N_{o}}\right) \quad \forall n \forall k \\
& \phi_{n, k}+\sum_{m \in M_{n}} \phi_{m, k} \leq 1 \quad \forall n \forall k \\
& 0 \leq \phi_{n, k} \leq 1 \quad \forall n \forall k \\
& \sum_{k \in C_{n}} \psi_{n, k} \leq 1 \quad \forall n
\end{array}
$$

where $r_{n, k}$ is the achievable link data rate where $B$ is the channel bandwidth, $N_{o}$ the noise power, and $P_{n, k}$ the received signal power for link $n$ when using channel $k$. The set of available channels for link $n$ is denoted as $C_{n}$. Eq. (7b) ensures the interference constraints, i.e., in each time slot, at most one secondary link can access the medium among the interfering links at any given time instant. Eq. (7d) indicates that each secondary node can access only one channel within a time slot.

Although P1 can provide an optimal solution for a given set of available channels $\left\{C_{n}\right\}$, it is not feasible to find an optimal solution for the following two reasons: P1

- involves both integer and continuous variables, rendering it mixed-integer nonlinear programming (MINLP) problem, which is NP-hard [26], and

- assumes the set of available channels $\left\{C_{n}\right\}$ known to secondary network, while they depend on the secondary users' sensing strategy (e.g., which and how many channels to sense) and instantaneous availability of the channels.

Therefore, instead of directly maximizing the network throughput, we analyze the sensing-access tradeoff in Section $\mathrm{V}$ and propose distributed spectrum sharing algorithms in Section VI.

\section{Sensing-Access Tradeoff}

We first introduce a new concept of $(\alpha, \beta)$-contention spectrum sharing, with which we analyze the sensing-access tradeoff. Understanding the sensing-access tradeoff will provide useful insights into throughput maximization problem as the spectrum-sensing overhead and the channel-access time are the two main factors that determine the achievable throughput.

Definition 2. ( $(\alpha, \beta)$-CONTENTION SPECTRUM SHARING) A spectrum-sharing policy $\Lambda$ achieves $(\alpha, \beta)$-contention if at least $\beta \in(0,1)$ fraction of secondary links in the network have equal to, or less than, $\alpha$ interfering links that have been assigned the same channel within a time slot. $\Lambda^{(\alpha, \beta)}$ represents a spectrum-sharing policy that satisfies $(\alpha, \beta)$ contention spectrum sharing.
According to the above definition, $(\alpha, \beta)$-contention spectrum sharing provides the following lower-bound on the achievable airtime share of secondary links:

$$
\begin{aligned}
& \operatorname{Pr}\left(M_{n}\left(\Lambda^{(\alpha, \beta)}\right) \leq \alpha\right) \geq \beta \\
\Longleftrightarrow & \operatorname{Pr}\left(\phi_{n}\left(\Lambda^{(\alpha, \beta)}\right) \geq \frac{T_{D}}{\tau} \cdot \frac{1-\delta}{\alpha+1}\right) \geq \beta \quad \forall \Lambda^{(\alpha, \beta)} .
\end{aligned}
$$

Eqs. (2) and (8) indicate that, for given $\beta$, the feasibility of $(\alpha, \beta)$-contention spectrum sharing depends on the number of interfering links that have been assigned the same channel. The number of interfering links can be reduced by sensing more channels at the cost of increased sensing time, and sharing them in a non-overlapping manner. We capture this interesting tradeoff between sensing and access via improper list coloring as we discuss next.

\section{E. Relationship with Improper List-coloring Problem}

In the contention-aware spectrum sharing problem, one can view the list of available channels (identified via sensing) at the secondary links as the list of colors $\left(L_{n}\right)_{n \in G_{c}}$ in the conflict graph $G_{c}$. The problem then becomes equivalent to the improper list-coloring problem. ${ }^{3}$ A graph $G=(V, E)$ is called $(c, \alpha)$-choosable (or c-choosable with impropriety $\alpha$ ), if for every (color) list assignment $L$ where $\left|L_{v}\right| \geq c \forall v \in V$, there exists $L$-coloring of $G$ such that each vertex of $G$ has at most $\alpha$ neighbors with the same color as its own [24]. Therefore, an $(\alpha, \beta)$-contention spectrum sharing is feasible if the conflict graph $G_{c}$ is $(c, \alpha)$-choosable where $c$ is the minimum number of available channels at the secondary nodes, i.e., $c \triangleq \min _{n \in V_{c}}\left\{\left|C_{n}\right|\right\}$, where $C_{n} \subseteq \mathcal{K}$ is the set of available channels at secondary link $n$.

\section{Sensing Requirements for Spectrum Sharing: A GRAPH-THEORETIC ANALYSIS}

We now consider the minimum requirements of channel probing for the $(\alpha, \beta)$-contention spectrum sharing, where $\alpha$ can be interpreted as the desired level of airtime share. We first study the distribution of the number of neighboring links under a point Poisson distribution, and derive the minimum number of available channels required at each secondary link for $(\alpha, \beta)$-contention spectrum sharing using the properties of improper/defective list-coloring [24].

\section{A. Distribution of Number of Neighbors}

Before analyzing the sensing requirement to achieve $(\alpha, \beta)$ contention, we need to understand the distribution of the number of neighboring secondary links. Assuming point Poissondistributed secondary links, the number of neighboring links of an arbitrary secondary link in the network follows the Poisson distribution as:

$$
N \sim \operatorname{Poi}\left(k ; \lambda \pi R_{I}^{2}\right),
$$

where $\lambda$ is the average secondary links density and $R_{I}$ the secondary interference range.

Then, the c.d.f. of the number of neighbors can be expressed as:

$$
F_{N}=\frac{\Gamma(\lfloor k+1\rfloor, \lambda)}{\lfloor k\rfloor !} \quad k \geq 0
$$

\footnotetext{
paper.

${ }^{3}$ The terms color and channel will be used interchangeably throughout this
} 


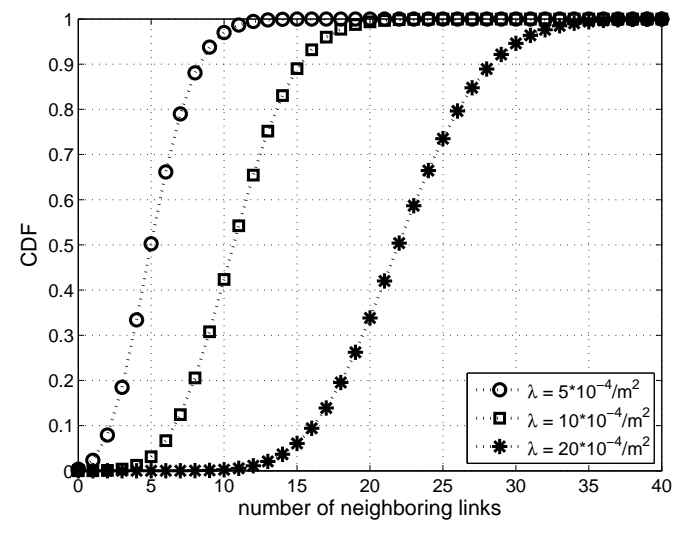

Fig. 2. The c.d.f. of number of neighbors under different link densities $\lambda=[500,1000,2000] / \mathrm{km}^{2}$. The interference range is set to $R_{I}=60 \mathrm{~m}$.

where $\Gamma(\cdot, \cdot)$ is the incomplete Gamma function and $k$ the number of neighboring links. Fig. 2 plots the c.d.f. of the number of neighboring links under different average link densities. Intuitively, the sensing requirement for $(\alpha, \beta)$-contention sharing depends on the maximum number of neighboring links in the network. However, due to the heavy-tailed nature of a Poisson distribution, $\operatorname{Pr}(k=\infty) \neq 0$, and thus, the worst-case analysis, i.e., $\beta=1$, is uninteresting and may not provide any useful insight in understanding the sensing-access tradeoff. We thus define $\beta$-maximal degree $(\beta<1)$ and consider it as the maximum number of neighboring links for a given network topology.

Definition 3. ( $\beta$-MAXIMAL DEGREE) The $\beta$-maximal degree of the secondary network is defined as:

$$
N_{\beta} \triangleq \sup \left\{N: F_{N}(\lambda) \leq \beta\right\},
$$

where $\lambda$ is the average secondary link density and $\beta \in(0,1)$ is a pre-defined constant (e.g., $\beta=0.95$ ).

From now, we consider the $\beta$-maximal degree as the maximum number of neighboring links of the network and study the sensing/probing requirements to achieve $(\alpha, \beta)$-contention sharing.

\section{B. Sensing Strategy for $(\alpha, \beta)$-contention Spectrum Sharing}

To achieve a longer channel-access time, i.e., small $\alpha$, the secondary users need to discover more available channels at the cost of more sensing time. Thus, the number of available channels for $(\alpha, \beta)$-spectrum sharing is an important performance metric. Therefore, we derive the minimum number of channels to be sensed for $(\alpha, \beta)$-contention channel assignment. Given an interference graph $G_{c}$, the problem can be formally stated as:

$$
\begin{array}{ll}
\text { Minimize } & \max _{n \in V_{c}}\left\{\left|C_{n}\right|\right\} \\
\text { subject to } & { }^{\exists} \Lambda^{(\alpha, \beta)} \text { s.t. } \operatorname{Pr}\left(M_{n}\left(\Lambda^{(\alpha, \beta)}\right) \leq \alpha\right) \geq \beta \quad \forall n .
\end{array}
$$

We prove the minimal sensing requirement for $(\alpha, \beta)$ contention spectrum sharing as follows.

Lemma 1. Assume a secondary network is denoted as a conflict graph $G_{c}$. Then, for $(\alpha, \beta)$-contention spectrum sharing, each secondary link $n \in V\left(G_{c}\right)$ must satisfy the following requirement for the number of available licensed channels:

$$
c \geq\left\lceil\frac{1}{2}\left(N_{\beta}-2 \alpha+\sqrt{N_{\beta}^{2}+\alpha^{2}}\right)\right]^{+} \forall n,
$$

where $c \triangleq \min _{n \in V_{c}}\left\{\left|C_{n}\right|\right\},\lceil\bullet\rceil^{+} \triangleq \max (0,\lceil\bullet\rceil)$, and $N_{\beta}$ is the $\beta$-maximal degree of the network.

Proof. Let us define the maximal average degree of conflict graph $G_{c}$ as $d\left(G_{c}\right) \triangleq\left\{\frac{1}{|H|} \sum_{v \in H} d(v) \mid H \subseteq G_{c}\right\}$. Let $\bar{D}(m, \alpha) \in \mathbb{R}$ denote the upper bound such that every conflict graph of the maximum average degree less than $\bar{D}(m, \alpha)$ is $(m, \alpha)^{*}$-choosable. Theorem 3 in [27] showed that $\bar{D}(m, \alpha)=\frac{m(m+2 \alpha)}{m+\alpha} \forall m \geq 2, \forall \alpha \geq 0$. Therefore, every graph with the $\beta$-maximal degree $N_{\beta}<\frac{m(m+2 \alpha)}{m+\alpha}$ is feasible for $(\alpha, \beta)$-contention spectrum sharing where $m \in \mathbb{N}$ is the minimum number of available channels at each vertex (i.e., secondary link). Then, by solving the above inequality, we have $m>\varphi=\frac{1}{2}\left(N_{\beta}-2 \alpha+\sqrt{\left(N_{\beta}-2 \alpha\right)^{2}+4 \alpha N_{\beta}}\right)$. Therefore, a graph of $\beta$-maximal degree $N_{\beta}$ with $\alpha$-impropriety is $\lceil\varphi\rceil$-choosable. Therefore, Eq. (12) holds.

Fig. 3 plots the required number of available channels in Eq. (12), which increases with network density.

\section{Achievable Channel-Access Time under Limited Sensing}

The number of available channels at secondary links may in practice be limited for various reasons, such as hardware constraints [16] or location and time-dependent spectrum availability [28], [29], [30]. The following lemma shows the achievable degree of channel contention under a limited number of available channels.

Lemma 2. For a given $\beta \in(0,1),(\alpha, \beta)$-contention spectrum-sharing policy $\Lambda^{(\alpha, \beta)}$ is achievable where

$$
\alpha= \begin{cases}\left\lceil\frac{c^{2}-c N_{\beta}}{N_{\beta}-2 c}\right]^{+} & \text {if } \quad \frac{N_{\beta}}{2} \leq c<N_{\beta} \\ 0 & \text { if } c \geq N_{\beta},\end{cases}
$$

where $c$ is the minimum number of available channels at secondary links, i.e., $c \triangleq \min _{n \in V_{c}}\left\{\left|C_{n}\right|\right\}$. In case $c<\frac{N_{\beta}}{2}$, no feasible solution for $\Lambda^{(\alpha, \beta)}$ is guaranteed.

Proof. Assume a conflict graph $G_{c}$ with $\beta$-maximal degree $N_{\beta}$. When $c \geq N_{\beta}$, at least $\beta$ fraction of nodes (i.e., secondary links) in the conflict graph have more colors than the number of its neighbors, and thus $(0, \beta)$-contention spectrum sharing can be achieved. When $\frac{N_{\beta}}{2} \leq c \leq N_{\beta}$, $(\alpha, \beta)$-contention spectrum sharing is achievable where $\alpha>\frac{c^{2}-c N_{\beta}}{N_{\beta}-2 c}$ (this can be shown by following similar procedures in the proof of Theorem 3 in [27]). Therefore, the result follows.

\section{Impact of Network Density on Sensing Requirement}

The following theorem shows that the sensing requirement for $(\alpha, \beta)$-contention spectrum sharing increases linearly with the secondary network density.

Theorem 1. Let $c$ denote the number of available channels required to achieve $(\alpha, \beta)$-contention spectrum sharing for given $\alpha$ and $\beta$. Then, for fixed $R_{I}$, we have

$$
c=O(\lambda), \quad \text { for } \lambda \geq \frac{5}{\pi R_{I}^{2}} .
$$




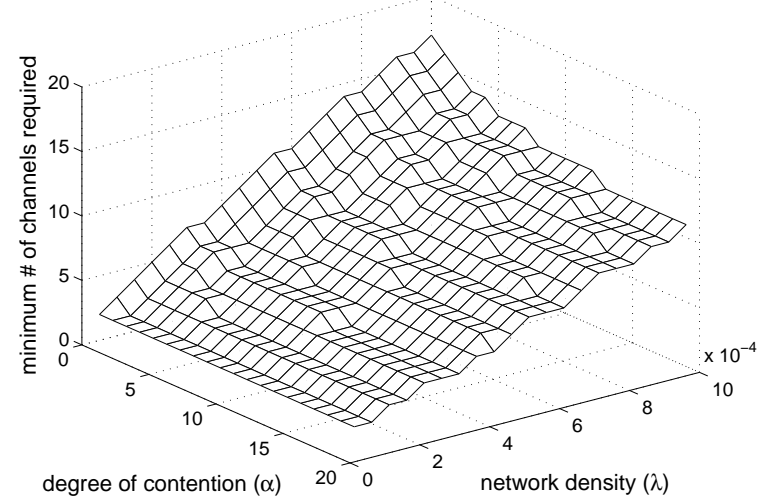

Fig. 3. Minimum number of available channels required to achieve $(\alpha, \beta)$ contention spectrum sharing almost linearly increases as either $\alpha$ (degree of channel contention) decreases or $\lambda$ (secondary link density) increases. The secondary interference range $R_{I}$ and $\beta$ are set to $60 \mathrm{~m}$ and 0.95 , respectively.

Proof. When the average number of neighboring links is large, i.e., $\lambda \pi R_{I}^{2}(\geq 5)$, the Poisson distribution of the number of neighboring links can be accurately approximated as a normal distribution [31]. Thus, by the definition in Eq. (11), we have $\beta=1-Q\left(\frac{N_{\beta}-\lambda \pi R_{I}^{2}}{\sqrt{\lambda \pi} R_{I}}\right)$ where $Q(x)=\frac{1}{\sqrt{2 \pi}} \int_{x}^{\infty} e^{-t^{2} / 2} \mathrm{~d} t$. Hence, $N_{\beta}=Q^{-1}(1-\beta) \sqrt{\lambda \pi} R_{I}+\lambda \pi R_{I}^{2}$. From Eq. (12), for a fixed interference range $R_{I}$, we have

$$
\begin{aligned}
c & \geq \frac{1}{2}\left(N_{\beta}-2 \alpha+\sqrt{N_{\beta}+\alpha^{2}}\right) \\
& \geq Q^{-1}(1-\beta) \sqrt{\lambda \pi} R_{I}+\lambda \pi R_{I}^{2}-2 \alpha \\
& =O(\lambda) .
\end{aligned}
$$

Thus, Eq. (13) holds.

Theorem 1 shows that the minimum number of available channels for $(\alpha, \beta)$-contention spectrum sharing increases at most linearly with the network density $\lambda$ (and thus $N_{\beta}$ ), as can be seen in Fig. 3. Therefore, the secondary link density is a critical factor in determining the minimum number of available channels to achieve $(\alpha, \beta)$-contention spectrum sharing.

Corollary 1. For fixed network density $\lambda$, we have

$$
c=O\left(P_{o}^{2 / \gamma}\right),
$$

where $P_{o}$ is the transmission power of the secondary user and $\gamma$ is the path-loss exponent (e.g., $\gamma=4)$.

Proof. Let $\eta$ denote the carrier-sensing threshold. Then, from Eq. (1), it can be approximated by $\eta \approx P_{o}\left(\frac{d_{o}}{R_{I}}\right)^{\gamma} \hat{X}$ where $R_{I}$ is the interference range and $\widehat{X}$ the average shadow fading gain, i.e., $\widehat{X}=\mathbb{E}\left[10^{\frac{X}{10}}\right]$. As a consequence, it can be easily shown that $R_{I} \approx G P_{o}^{1 / \gamma}$ where $G=d_{o}^{-1}\left(\frac{\widehat{X}}{\eta}\right)^{1 / \gamma}$ is a constant. According to Eq. (13b), $c=O\left(R_{I}^{2}\right)=G^{2} O\left(P_{o}^{2 / \gamma}\right)$. Therefore, Eq. (14) follows.

Corollary 1 indicates that, for fixed network density, the transmission power of secondary users influences the sensing strategy for achieving $(\alpha, \beta)$-contention sharing. This confirms a common intuition: the stronger the transmission power, the higher the link data rate, but severer the channel contention due to the increased interference range, and vice versa.
While the above analytical findings provide useful insights on the sensing-access tradeoff, the achievable network throughput also depends on how efficiently the thus-discovered available channels are shared in the channel assignment phase ( $T_{A}$ in Fig. 1). Therefore, we propose distributed algorithms for spectrum sharing that resolves channel contention among neighboring links, while exploiting heterogeneous channel conditions as much as possible to maximize the network throughput.

\section{Contention-Aware Distributed SPECTRUM-SHARING ALGORITHMS}

The optimization problem P1 developed in Section IV-C suffers from (i) computational complexity due to its NPhardness and (ii) lack of scalability because it requires a central node for spectrum sharing. We therefore seek low-complexity distributed spectrum sharing protocols. Specifically, we propose distributed spectrum sharing algorithms and present them in the order of increasing implementation complexity and optimality.

The first algorithm (i.e., Local Best) is non-cooperative where each secondary node independently selects a color (channel) among its list of available colors, which will be used as a performance benchmark in Section VII. The next two algorithms-Color-Switch and Color-Exchange-are cooperative channel assignment, allowing secondary nodes to adaptively change the color after collecting the color information from its neighbors. Then, we propose Smart Share, which adaptively executes one of the two cooperative algorithms for the best channel assignment.

\section{A. ALG1: Local Best}

In Local Best, secondary nodes select the best channel from their list $C_{n}$ of available channels, based on the expected reward (i.e., average data rate). That is, the secondary nodes on link $n$ select the channel $i^{*}$ such that $i^{*}=\arg \max _{i \in C_{n}}\left\{r_{n, i}\right\}$. Local Best is simple and easy to implement, but it does not have any color conflict-resolution mechanism, and may thus suffer from the channel-contention overhead (see Section VII). This motivates the design of cooperative algorithms that adjust color based on the color information of neighboring nodes.

\section{B. ALG2: Color-Switch}

In this algorithm, secondary nodes switch color after a local search for the color information of neighboring nodes. For the local search, a node with the smaller ID of each link will be the representative node; only the representative nodes will participate in the channel-assignment process. Algorithm 1 shows the procedure of exchanging the color information among neighboring secondary nodes. Initially, the secondary nodes select a channel by executing Local Best. Then, they share the color information with neighboring nodes. This can be done by adopting a random backoff in the IEEE 802.11 MAC protocol. ${ }^{4}$ Once the backoff timer expires, the secondary node broadcasts a GETCOLOR message to its neighbor (interfering) nodes for their color information; the receivers of this

\footnotetext{
${ }^{4}$ Secondary nodes will set a timer $t_{\text {Backoff }}$ by selecting a number uniformly distributed between $[0, W-1]$, where $W$ is the backoff window size.
} 


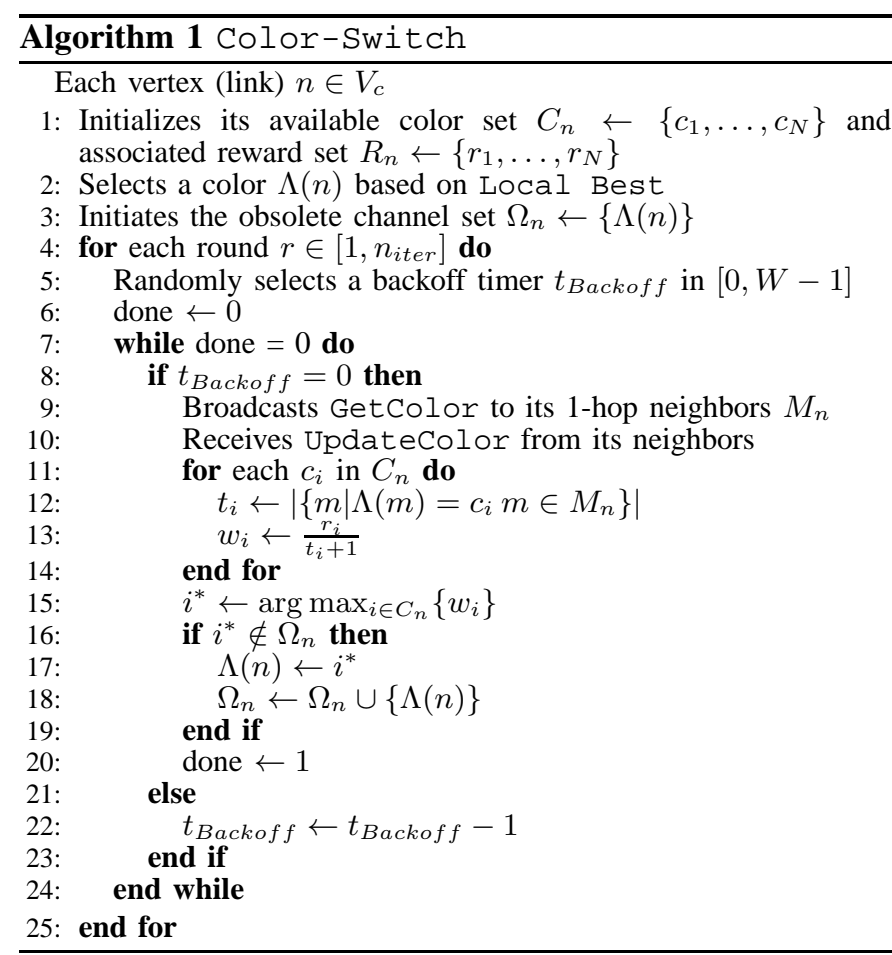

message will respond with an UPDATECOLOR message, which includes the available channel list with the associated channelcondition information and their current channel selection. The MAC frame formats are provided in Fig. 4. Then, based on the collected color information, the initiating secondary node switches to (or stays at) the best channel among those that have not been selected before (i.e., $C_{n} \backslash \Omega_{n}$ ) for fast convergence in channel selection. This algorithm requires $O(\Delta)$ time for data collection where $\Delta$ is the maximum degree of the conflict graph. This color information exchange process among neighboring nodes is repeated for $n_{i t e r}$ times where secondary nodes perform Color-Switch in each round $r$. Since each $\{$ LINK, COLOR $\}$ pair can be picked at most once for fast convergence, the number of rounds is upper bounded by the maximum number of available channels at secondary nodes, i.e., $n_{\text {iter }} \leq C_{\max }=\max \left\{\left|C_{n}\right|\right\}_{n \in V_{c}}$. Therefore, the time complexity of this algorithm is $O\left(\Delta \cdot\left|V_{c}\right| \cdot C_{\max }\right)$. Note that the protocol-based actual time overhead of this algorithm will be analyzed in Section VII.

The main drawback of Color-Switch is that it may not be able to escape from a local optimum due to the sequential execution of the algorithm. This motivates the following algorithm that allows two neighboring nodes to swap their color simultaneously.

\section{ALG3: Color-Exchange}

In this algorithm, secondary nodes attempt to maximize the reward by exchanging color information with one of their neighbor nodes. Algorithm 2 shows the color-exchange procedure (also see Fig. 4). Secondary nodes first collect the color information of neighbor nodes using GETCOLOR/UPDATECOLOR messages, then choose the best candidate neighbor for color exchange. Once a target neighbor is picked, the initiating secondary node will send a

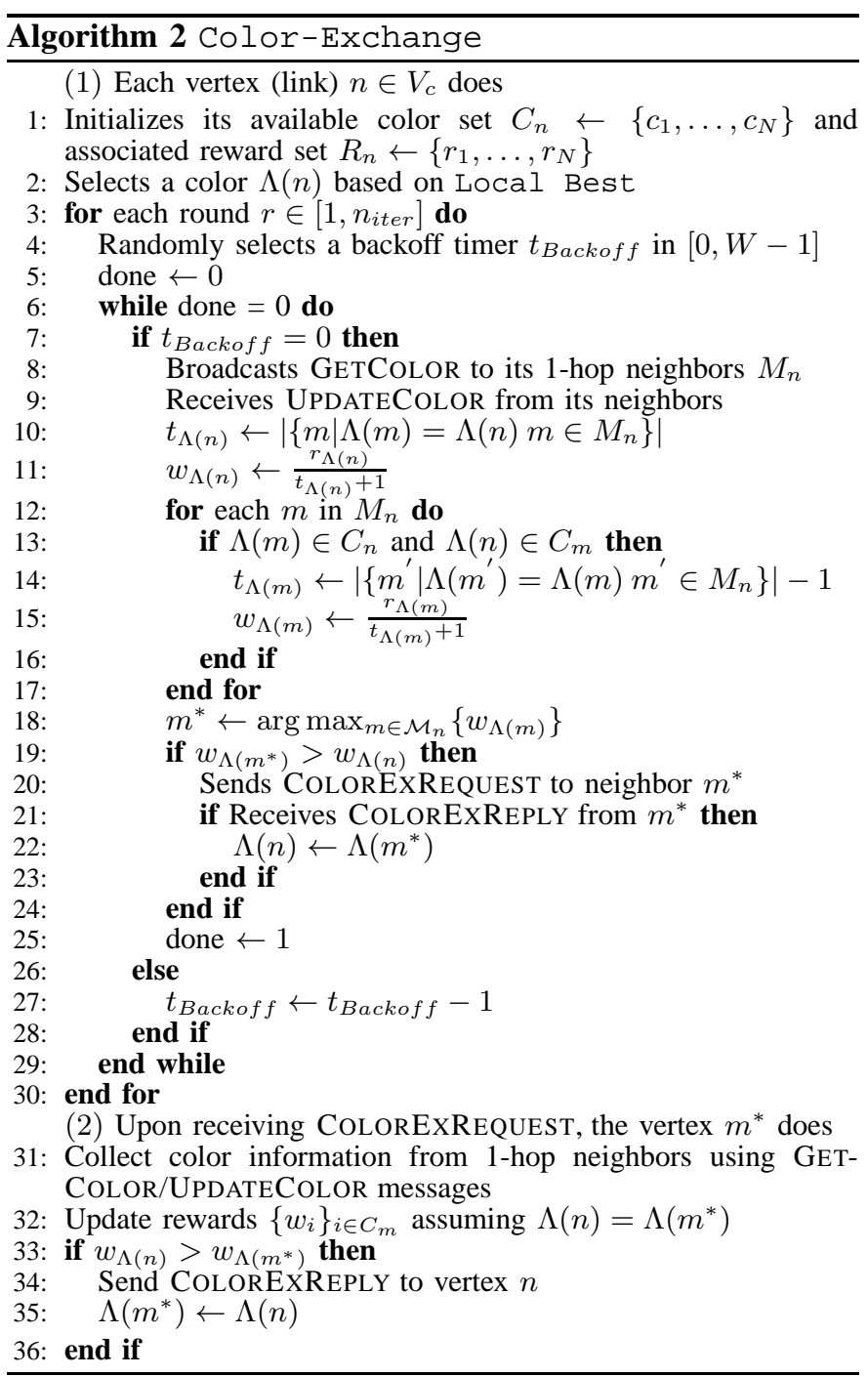

COLOREXREQUEST message for color-information exchange; the receiver of this message agrees to the exchange only when it is beneficial (increasing reward) to itself. In other words, in Color-Exchange, the exchange must be mutually beneficial to both participating secondary nodes. If the receiving node agrees to the exchange, it will respond with a COLOREXREPLY message and then switch to the suggested color; otherwise, it will not respond. Upon receiving the COLORREPLY message within a certain time period, the initiating secondary node will also switch color.

\section{ALG4: Smart Share}

This algorithm allows a secondary node to perform either Color-Switch or Color-Exchange, whichever performs better (i.e., provides a higher increase in total reward). The initiating node collects the color information via the GETCOLOR/UPDATECOLOR mechanism and then calculates the best possible local improvement when it performs Color-Switch and Color-Exchange, respectively. If exchanging color information with one of its neighbors improves its own reward more than switching its own color, the initiating node proceeds with COLOREXRE- 


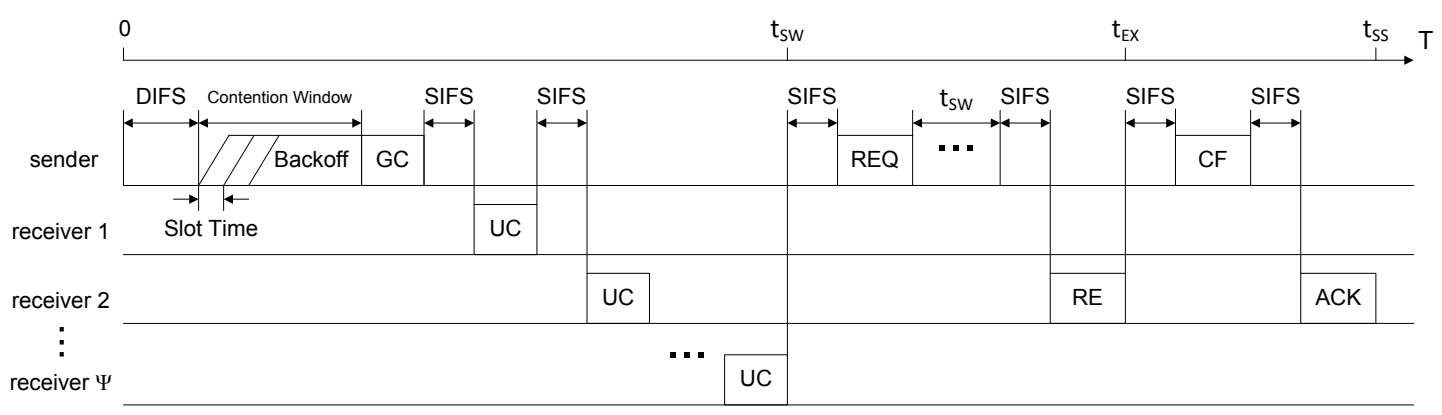

GC: GETCOLOR UC: UPDATECOLOR REQ: COLOREXREQUEST RE: COLOREXREPLY CF: EXCONFIRM ACK: EXACK

Fig. 4. The protocol behavior for exchanging the color information: cooperative channel-assignment algorithms, i.e., Color-Switch, Color-Exchange, and SmartShare require the color information exchange overheads of $t_{S W}, t_{E X}$, and $t_{S S}$, respectively.

\begin{tabular}{|c|c|c|c|c|c|c|c|}
\hline $\begin{array}{c}\text { octets: } 2 \\
\text { Frame } \\
\text { Control }\end{array}$ & Duration & RA1 & RA2 & $\cdots$ & RA16 & TA & FCS \\
\hline
\end{tabular}

(a) GETCOLOR

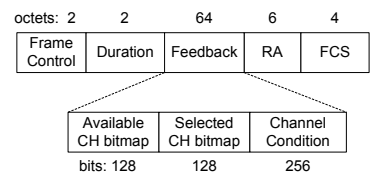

(b) UPDATECOLOR

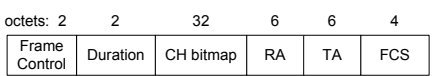

(d) ColorExRequest

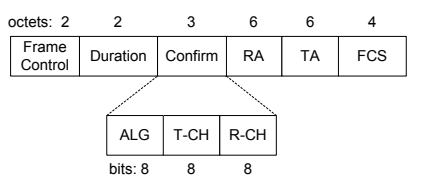

(c) EXCONFIRM
Fig. 5. MAC frame formats used by the proposed algorithms.

QUEST/COLOREXREPLY messages. After receiving the feedback from a neighbor, the initiating node finalizes the procedure by notifying the neighboring node (i.e., the one considered for exchanging color information) the adopted algorithm via EXCONFIRM/EXACK messages, as shown in Fig. 4.

\section{E. MAC Frame Formats for the Proposed Algorithms}

To realize the proposed algorithms, we design a medium access protocol on the basis of the IEEE 802.11 DCF. Fig. 4 shows the overall protocol behavior for exchanging colorinformation; the MAC frame formats used for the proposed algorithms are shown in Fig. 5. The formats of GETCOLOR and UPDATECOLOR are shown in Figs. 5(a) and 5(b), respectively. The GETCOLOR frame can use either the broadcast address or list of receivers (RAs) (maximum 16). The UPDATECOLOR frame contains the bitmap of both available and selected channels at the sender; a maximum of 128 channels are supported. It also contains the channel condition (i.e., achievable average data rate) information of the available channels to exploit multi-channel diversity. Although we assume that each secondary node accesses at most one channel at a time, our algorithms can be easily extended to support the use of multiple channels. The format of COLOREXREQUEST contains channel bitmap information to specify the channels for exchange with the receiver, as shown in Fig. 5(d). The EXCONFIRM format is only for Smart Share and specifies the algorithm, i.e., Color-Switch or Color-Exchange, chosen by the initiating node, and if Color-Exchange is selected, it conveys the channels for exchange, i.e., T-CH and $\mathrm{R}-\mathrm{CH}$, as depicted in Fig. 5(c). The COLOREXREPLY and EXACK use the same frame format with different ACK bitmap subfields.

\section{PERformance Evaluation}

In this section, we first analyze the channel sensing and assignment overheads of the proposed algorithms. We then evaluate their performance under various network conditions.

\section{A. Analysis of Protocol Overheads}

Secondary nodes must suspend data transmission during the channel sensing and assignment periods. Thus, the time spent on the sensing and assignment should be considered as a performance loss. Here we quantify these time overheads (i.e., $T_{P}$ and $T_{A}$ ) of channel-assignment algorithms based on the proposed MAC protocols.

1) Channel-Sensing Overhead $\left(T_{P}\right)$ : To probe a channel, a secondary node first senses its (un)availability using feature detection for the duration of $T_{s}$, and then measures its condition for the duration of tMEASURE. These sensing and measurement operations will be repeated for the entire set of channels to be sensed (i.e., $S_{n}$ ). Therefore, the total sensing/probing time can be calculated as:

$$
T_{P}\left(n_{s}\right)=\left[T_{s}+t M E A S U R E\right] \cdot n_{s},
$$

where $n_{s}$ is the number of channels to be sensed, i.e., $\left|S_{n}\right|$. According to the system parameters listed in Table I, sensing a single channel takes $24 \mathrm{~ms}$ for the field sync detector for a DTV signal [23], which translates to $1.2 \%$ of the time slot $(\tau)$.

2) Channel-Assignment Overhead $\left(T_{A}\right)$ : The time overhead of channel assignment $T_{A}$ depends on the underlying algorithm. For example, $T_{A}=0$ for a non-cooperative channel assignment algorithm (ALG1) since it does not require messages to be exchanged for channel assignment. On the other hand, cooperative algorithms (ALG2-4) require message exchanges with neighboring nodes and the time overhead depends on the network density, especially the number of neighboring links.

Based on Fig. 4, the average time overheads of Color-Switch, Color-Exchange, and Smartshare can be approximated as shown in Eq. (16). Note that the time overhead of SmartShare in Eq. (18) depends on the algorithm selected by the initiating node (i.e., Color-Switch 


$$
\begin{aligned}
& \mathbb{E}\left[T_{A}^{S w}(\Psi)\right] \approx\left[t D I F S+(\Psi+1) \cdot t S I F S+t G E T_{-} C O L O R+\Psi \cdot t \text { UPDATE_COLOR }+\bar{T}_{\text {back }}\right] \cdot(\Psi+1) \cdot n_{\text {iter }}=t_{S W} \cdot(\Psi+1) \cdot n_{\text {iter }} . \\
& \mathbb{E}\left[T_{A}^{\mathrm{Ex}}(\Psi)\right] \approx\left[2 \cdot t_{S W}+2 \cdot t S I F S+t E X \_R E Q U E S T+t E X \_R E P L Y\right] \cdot(\Psi+1) \cdot n_{\text {iter }}=t_{E X} \cdot(\Psi+1) \cdot n_{\text {iter }} . \\
& \mathbb{E}\left[T_{A}^{S S}(\Psi)\right] \approx\left[\rho \cdot t_{S W}+(1-\rho) \cdot\left(t_{E X}+t E X \_C O N F I R M+t E X \_A C K+2 \cdot t S I F S\right)\right] \cdot(\Psi+1) \cdot n_{\text {iter }}=\left[\rho \cdot t_{S W}+(1-\rho) \cdot t_{S S}\right] \cdot(\Psi+1) \cdot n_{i t e r} \cdot(1
\end{aligned}
$$

\begin{tabular}{|c|c|c|}
\hline Parameter & Value & Comments \\
\hline$T_{s}$ & $24 \mathrm{~ms}$ & Channel-sensing duration \\
\hline $\bar{T}_{\text {back }}$ & $72 \mu \mathrm{s}$ & Average random backoff period \\
\hline$t D I F S$ & $34 \mu \mathrm{s}$ & DIFS time \\
\hline$t S I F S$ & $16 \mu \mathrm{s}$ & SIFS time \\
\hline tMEASURE & $146 \mu \mathrm{s}$ & $2 \times$ SIFS + RTS + CTS \\
\hline$t G E T \_C O L O R$ & $172 \mu \mathrm{s}$ & GETCOLOR duration \\
\hline tUPDATE_COLOR & $132 \mu \mathrm{s}$ & UPDATECOLOR duration \\
\hline$t E X \_R E Q U E S T$ & $96 \mu \mathrm{s}$ & COLOREXREQUEST duration \\
\hline$t E X \_R E P L Y$ & $44 \mu \mathrm{s}$ & COLOREXREPLY duration \\
\hline tEX_CONFIRM & $56 \mu \mathrm{s}$ & EXCONFIRM duration \\
\hline$t E X \_A C K$ & $44 \mu \mathrm{s}$ & EXACK duration \\
\hline
\end{tabular}

TABLE I

THE SYSTEM PARAMETERS FOR OUR PROPOSED ALGORITHMS

or Color-Exchange), where $\rho$ is the estimated ratio at which the node decides to adopt Color-Switch over Color-Exchange.

Eqs. (16), (17), and (18) indicate that the average time spent on channel assignment depends on (i) the average node density $\Psi$ and (ii) the number of iterations $n_{\text {iter }}$ for message exchanges, which is a design parameter. For example, assuming $\Psi=4, n_{\text {iter }}=2$, and $\rho=0.985,{ }^{5}$ the average channelassignment times are $\mathbb{E}\left[T_{A}^{\mathrm{Sw}}\right]=10.18 \mathrm{~ms}, \mathbb{E}\left[T_{A}^{\mathrm{Ex}}\right]=22.08 \mathrm{~ms}$, and $\mathbb{E}\left[T_{A}^{\mathrm{Ss}}\right]=10.38 \mathrm{~ms}$, which correspond to $0.51 \%, 1.1 \%$, and $0.52 \%$ of a time slot, respectively. Note that these channelassignment overheads are shorter than the time required to probe a single channel, $T_{S}$, implying that the time overhead of the proposed algorithms would not significantly affect the system performance, as will be evident in our simulation results.

3) Airtime Share $(\phi)$ : Considering the time overheads analyzed above, the expected airtime share of secondary nodes under policy $\Lambda$ can be computed based on Eqs. (2), (15), (16), (17) and (18), as follows:

$$
\begin{aligned}
& \phi_{n}\left(\Psi, n_{s}, n_{\text {alg }}, \Lambda\right) \\
& \quad=\left[1-\frac{T_{P}\left(n_{s}\right)+T_{A}\left(\Psi, n_{\text {alg }}\right)}{\tau}\right]^{+} \cdot \frac{1-\delta}{M_{n}(\Lambda)+1} \quad \forall n,
\end{aligned}
$$

where

$$
T_{A}\left(\Psi, n_{\text {alg }}\right) \triangleq \begin{cases}\mathbb{E}\left[T_{A}^{\mathrm{Sw}}(\Psi)\right] & \text { if } n_{\text {alg }} \in\{2\} \\ \mathbb{E}\left[T_{A}^{\mathrm{Ex}}(\Psi)\right] & \text { if } n_{\text {alg }} \in\{3\} \\ \mathbb{E}\left[T_{A}^{\mathrm{SS}}(\Psi)\right] & \text { if } n_{\text {alg }} \in\{4\} \\ 0 & \text { otherwise, }\end{cases}
$$

where $n_{a l g}$ is the index for the channel-assignment algorithms. The protocol-related system parameters are listed in Table I, where the parameter values are derived based on the MAC frame formats in Fig. 5 assuming the IEEE 802.11a OFDM PHY [32] for modulation and channel coding.

\footnotetext{
${ }^{5}$ This value was found from the Monte Carlo simulation over $10^{5}$ repetitions.
}

TABLE II

THE PARAMETERS USED IN THE SIMULATION

\begin{tabular}{|l|l|l|}
\hline Parameter & Value & Comments \\
\hline \hline$P_{o}$ & $25 \mathrm{~mW}$ & Transmit power \\
\hline$N_{o}$ & $5 \times 10^{-11} \mathrm{~mW}$ & Noise power \\
\hline$\gamma$ & 4 & Path-loss exponent \\
\hline$\sigma_{d B}$ & 5.5 & Shadowing dB-spread \\
\hline$\lambda$ & {$[100-2800] / \mathrm{km}^{2}$} & Avg. secondary link density \\
\hline$d_{i}$ & $20-40 \mathrm{~m}$ & Secondary link distance \\
\hline$R_{I}$ & $60 \mathrm{~m}$ & Interference range \\
\hline$\delta$ & 0.3 & Contention overhead \\
\hline$n_{\text {iter }}$ & 3 & Number of iterations \\
\hline$\tau$ & $2 \mathrm{~s}$ & Time slot duration \\
\hline$\beta$ & 0.95 & $\beta$-maximal degree \\
\hline
\end{tabular}

We consider a CRN in which primary and secondary users coexist in a $5 \times 5 \mathrm{~km}^{2}$ area. We assume that secondary links are randomly distributed following a point Poisson distribution with average density $\lambda$, and secondary link distances are uniformly distributed in $[20,40] \mathrm{m}$. We assume 5 primary transmitters randomly distributed in the area, each of which randomly selects and utilizes one of 10 licensed channels. The primary interference range is assumed to be $200 \mathrm{~m}$; any secondary nodes located within this range can detect the primary signal and will restrain from accessing the channel if a primary signal is detected. Throughout the simulation, we set the time-slot duration to $\tau=2 \mathrm{~s}$, considering the 2second channel detection time (CDT) in the IEEE 802.22 draft standard [23]. The shadow fading dB-spread is set to $\sigma_{d B}=5.5 \mathrm{~dB}$, typically assumed in IEEE 802.22 (i.e., rural areas) [23]. The channel-contention overhead is considered by setting $\delta=0.3$, which is the average overhead in channel access time due to contention in the IEEE 802.11b WLANs over different data rates [33]. We assume that the number of iterations for cooperative spectrum-sharing algorithms is fixed at $n_{\text {iter }}=3 .^{6}$ The simulation parameters are listed in Table II.

\section{B. Simulation Setup}

To demonstrate the benefits of our contention-aware algorithms, we evaluate the performance of the following distributed spectrum-sharing algorithms: (1) Local Best, (2) Color-Switch, (3) Color-Exchange, (4) SmartShare, and (5) Distributed Collaborative Max-Sum Bandwidth (D-CMSB). ${ }^{7} \mathrm{D}-\mathrm{CMSB}$ is a low-complexity distributed modification of the CMSB rule proposed in [34]. The basic idea of CMSB is to maximize the network sumbandwidth by letting each secondary node select the best

\footnotetext{
${ }^{6}$ Our simulation result indicates that Smartshare converges within 3 iterations over $90 \%$ of the time.

${ }^{7} \mathrm{D}-\mathrm{CMSB}$ performs channel selection simultaneously at secondary user nodes, instead of sequentially assigning a color to each node, as proposed in [34]. Thus, D-CMSB can be implemented using the same MAC protocol designed for Color-Switch, thus significantly reducing the time overhead.
} 


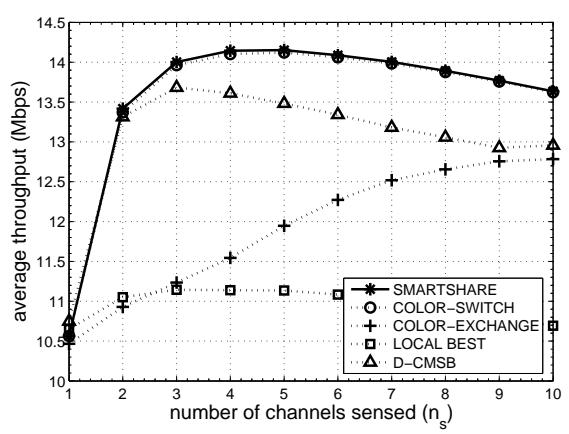

(a) Throughput

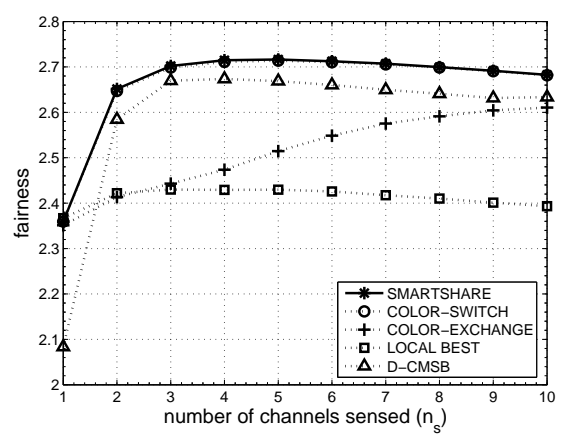

(b) Fairness

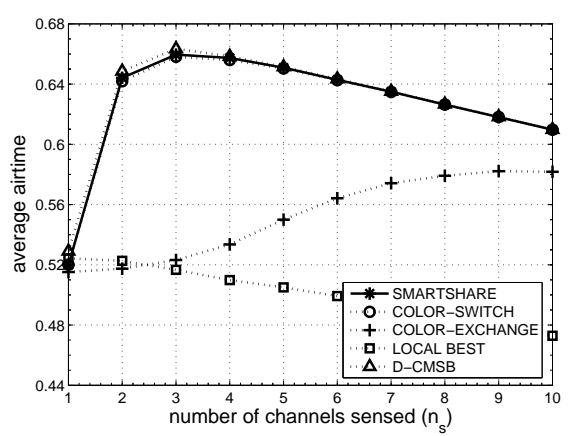

(c) Airtime

Fig. 6. Comparison of the testing schemes: The figures show that SmartShare outperforms the other testing algorithms in terms of (a) throughput, (b) fairness, and (c) airtime. The average secondary link density is set to $\lambda=500 / \mathrm{km}^{2}$.

channel considering both channel heterogeneity and conflict relations among neighboring nodes (see Section 3 of [34] for more details). The MAC protocols of Color-Switch can be used for exchanging color information for $\mathrm{D}-\mathrm{CMSB}$ and this algorithm will represent the performance of the conventional interference-free spectrum-sharing algorithms.

\section{Performance Metrics}

To evaluate the performance of the above-mentioned algorithms, we use the following three performance metrics.

1) Throughput: we measure the average achieved link throughput, i.e., $\frac{1}{\mid V_{c}} \sum_{n \in V_{c}} Z_{n}(\Lambda)$, of the algorithms.

2) Fairness: we measure the proportional fairness in spectrum sharing, i.e., $\frac{1}{\left|V_{c}\right|} \sum_{n \in V_{c}} \log \left(1+Z_{n}(\Lambda)\right)$. Note that we add 1 to the throughput $Z_{n}$ to avoid the argument of logarithm becoming 0 .

3) Airtime: we measure the average achieved timeshare at secondary nodes, i.e., $\frac{1}{\left|V_{c}\right|} \sum_{n \in V_{c}} \phi_{n}(\Lambda)$, of the algorithms.

Each simulation is performed on 20 random topologies, unless otherwise specified, and their averages are taken as the performance measures.

\section{Impact of Sensing Strategy}

We first compare the proposed contention-aware algorithms, and then compare them with the interference-free D-CMSB.

Fig. 6(a) plots the average network throughput achieved by the testing algorithms. We make three observations. First, as expected, cooperative algorithms outperform the noncooperative algorithm, i.e., Local Best, thanks to their ability to resolve channel contention and exploit channel heterogeneity. The throughput performance of Local Best decreases as the number of probing channels increases due to the increased sensing time overhead, whereas the performance of Color-Exchange improves with the number of channels. There is an exception: Local Best outperforms Color-Exchange with a small number of sensed channels (i.e., $n_{s}<3$ ) where the benefit from exchanging channelcondition information of Color-Exchange is negligible, while Local Best does not incur any time overhead.

Second, the achievable network throughput of SmartShare exceeds that of the other cooperative algorithms. The throughput of Smartshare exhibits concavity, indicating the tradeoff between the sensing overhead and the potential throughput gain, i.e., the throughput suffers with too small (large) a number of channels from channel contention (sensing overheads). Note that Color-Switch largely outperforms Color-Exchange due to the limited applicability of the latter (i.e., both secondary users must always agree on the exchange of the color information), while the former allows more flexibility in adjusting colors based on the neighbors' color information.

Third, two of our cooperative contention-aware algorithms, i.e., Color-Switch and SmartShare, outperform the interference-free $\mathrm{D}-\mathrm{CMSB}$, in spite of the fact that they achieve less total airtime than D-CMSB due to the channel contention overhead, as shown in Fig. 6(c). This is because of their sophisticated spectrum-sharing mechanisms that jointly consider the expected channel access time and heterogeneous channel conditions.

Fig. 6(b) shows that Smart Share achieves better fairness than the interference-free D-CMSB for all simulated scenarios. SmartShare achieves better fairness even with a small number of sensed channels, e.g., $n_{s} \leq 2$, where D-CMSB achieves a similar level of throughput, as shown in Fig. 6(a). This is because our contention-aware algorithms guarantee the secondary users' channel access in each time slot insofar as at least one available channel is discovered via sensing. By contrast, D-CMSB allows channel access to secondary users only when there is no interfering link assigned the same channel; otherwise, only one of the interfering nodes can access the channel.

Fig. 6(c) plots the average achieved channel access time (airtime) of secondary links. Interestingly, Smartshare achieves at least $98.3 \%$ of D-CMSB's airtime, in spite of the fact that SmartShare suffers from the channel-contention overhead, whereas D-CMSB does not experience any contention due to its interference-free channel assignment. However, Smartshare achieves better throughput and fairness as shown in Figs. 6(a) and 6(b), demonstrating its efficacy in sharing the discovered spectrum opportunities.

\section{E. Impact of Network Density}

We now investigate the impact of network density on the achievable network throughput with Smartshare. We ran simulations under average secondary link densities $\lambda$ ranging from $1 \times 10^{-4} / \mathrm{m}^{2}$ to $28 \times 10^{-4} / \mathrm{m}^{2}$, with the corresponding 


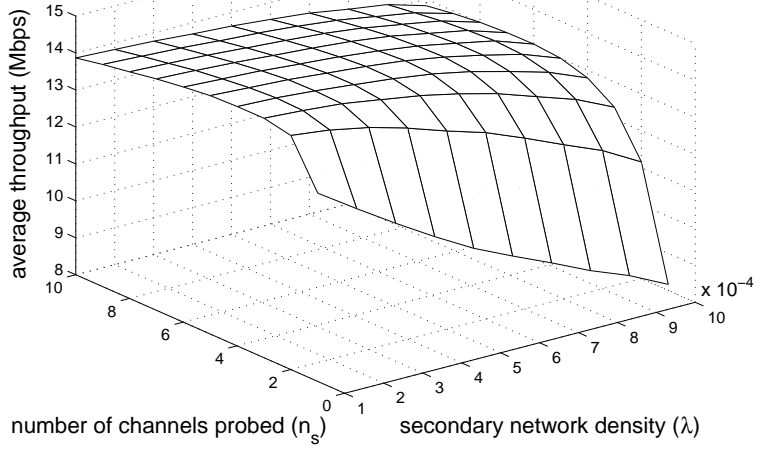

(a) Average link throughput

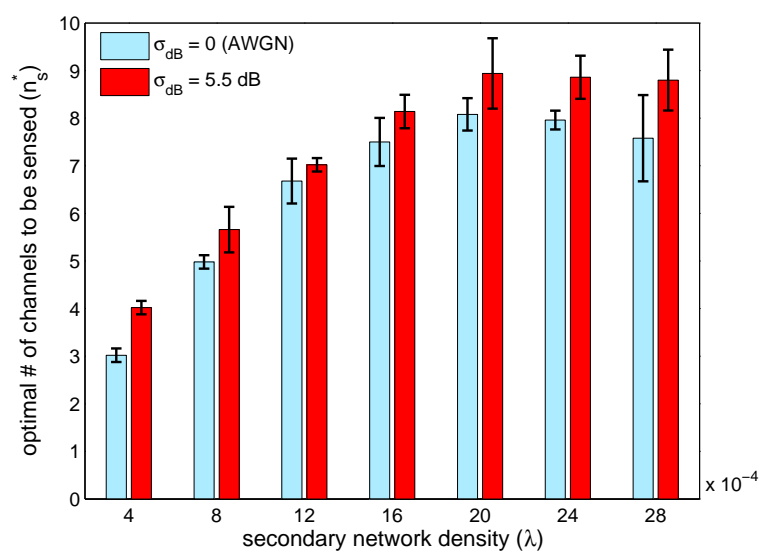

(b) Optimal spectrum sensing

Fig. 7. Impact of network density on throughput of Smart Share: (a) The average link throughput decreases as the average secondary link density ( $\lambda$ ) increases, regardless of the number of channels sensed $\left(n_{s}\right)$, and (b) the average and standard deviation of the optimal number of channels to be sensed $\left(n_{s}^{*}\right)$ for different secondary link densities. The results are obtained from simulations with 50 random topologies.

average number of neighboring links of 1.14 and 31.92 , respectively. Fig. 7(a) clearly shows that the number of channels to be sensed should be adapted to the network density to maximize the network throughput. The figure indicates that, by sensing an optimal number of channels for given network density, the throughput of Smart Share can be improved by up to $60 \%$ over the scheme that senses only a single channel.

Fig. 7(b) shows that the optimal number, $n_{s}^{*}$, of channels to be sensed varies with the secondary link density. It shows that channel heterogeneity, i.e., $\sigma_{d B}=5.5 \mathrm{~dB}$, results in sensing a larger number of channels than the homogeneous channel case, i.e., $\sigma_{d B}=0 \mathrm{~dB}$ (AWGN). This is because Smart Share can exploit heterogeneous channel conditions, and thus, the throughput gain from channel heterogeneity compensates for the increased sensing time overhead. Interestingly, $n_{s}^{*}$ starts to decrease as the number of neighboring links exceeds 22.8 , i.e., $\lambda=20 \times 10^{-4} / \mathrm{m}^{2}$, as the throughput gain from reducing the sensing time exceeds that from resolving channel-access contention among neighboring links.

\section{F. Impact of Shadow Fading}

We now evaluate the impact of heterogeneous channel conditions due to shadow fading on the performance of SmartShare. Fig. 8 shows the average link throughput in various shadow fading environments. The figure indicates that Smart Share achieves higher throughput with heterogeneous channels, i.e., $\sigma_{d B}>0$, than with homogeneous channels, i.e., $\sigma_{d B}=0$. An interesting observation is that the optimal number, $n_{s}^{*}$, of channels to be sensed increases as shadow fading becomes severer, i.e., higher $\mathrm{dB}$-spread $\sigma_{d B}$, thanks to SmartShare's ability to exploit heterogeneous channel conditions. Therefore, we can conclude that channel heterogeneity is also an importance factor in designing an optimal spectrum-sensing strategy.

\section{G. Impact of Channel Availability}

We study the impact of channel availability on the throughput performance of SmartShare. Fig. 9 plots the average secondary link throughput for various channel idle probabilities $p_{\text {idle }}$, i.e., the probability that no primary signal exists in the channel. The figure indicates that, when $p_{\text {idle }}$ is high, it is

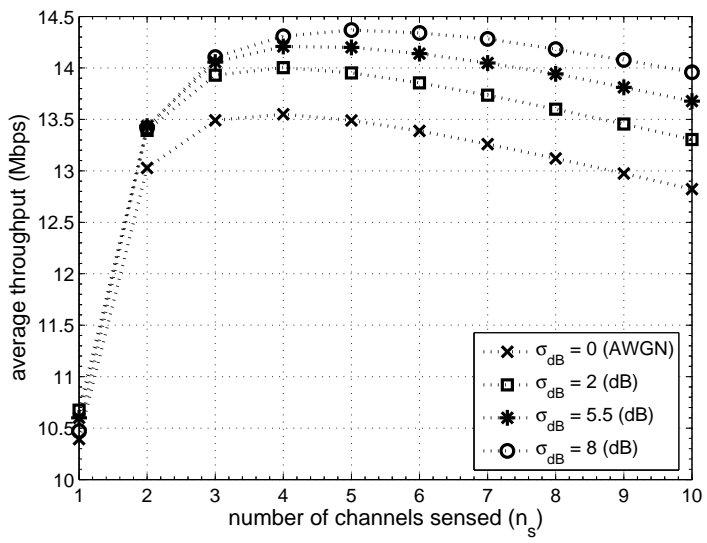

Fig. 8. Impact of shadow fading: Channel heterogeneity due to shadow fading encourages secondary nodes to sense more channels since the throughput gain from channel diversity can compensate for the throughput loss due to the sensing overhead. The secondary links density is set to $\lambda=500 / \mathrm{km}^{2}$.

sufficient to sense a smaller number of channels to maximize the throughput. However, as $p_{\text {idle }}$ decreases, it becomes desirable to sense more channels to discover available channels at the cost of sensing time overhead. Therefore, the channel availability significantly affects the achievable throughput, and must thus be considered in the design of a sensing strategy, along with other factors such as network density, transmission power, and channel heterogeneity. While we assume all the channels have same $p_{\text {idle }}$, a more sophisticated sensing strategy (e.g., [13], [15], [16]) can be used if channels have different $p_{\text {idle }}$ s. Although the problem of channel selection and ordering for sensing is an interesting problem, it is not within the scope of this paper.

\section{CONCLUSION}

An optimal spectrum-sensing scheme is of great importance to the maximization of secondary network performance. In this paper, we exploited the sensing-access tradeoff-between spectrum sensing overhead and channel access of secondary users - which has not been addressed adequately before. We formulated the secondary network throughput maximization 


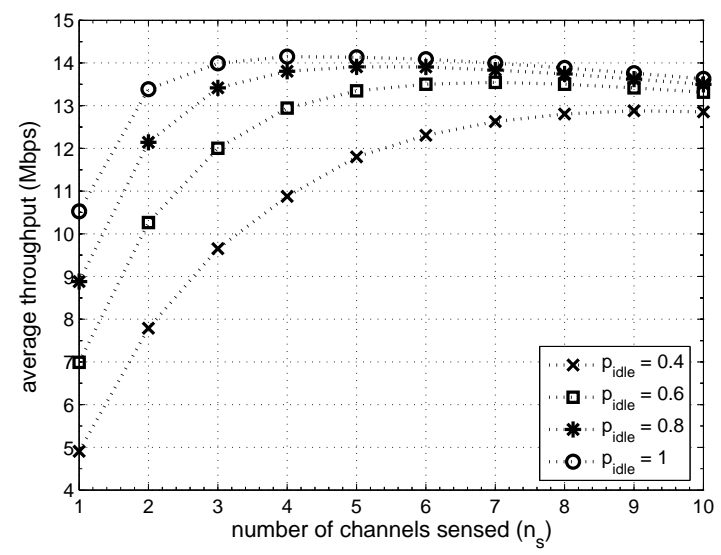

Fig. 9. Impact of channel availability: The channel idle probability influences the secondary network throughput. The secondary users need to sense a less number of channels with a high channel idle probability, and vice versa. The secondary links density is set to $\lambda=500 / \mathrm{km}^{2}$.

as MINLP, which turns out to be NP-hard. To analyze the sensing-access tradeoff, we introduced a new concept of $(\alpha, \beta)$-contention spectrum sharing, then used the improper list-coloring to derive the relationships between the channel contention requirement, the network density, and the transmission power level. We also proposed distributed contentionaware spectrum-sharing algorithms, including Smart Share, and described how to realize SmartShare in the 802.11 MAC protocol for its practical use and performance evaluation. Our in-depth simulation demonstrated the efficacy of SmartShare, and showed that it can enhance performance up to $60 \%$ by exploiting the sensing-access tradeoff.

\section{ACKNOWLEDGEMENT}

The work reported in this paper was supported in part by NSF under grants CNS-0519498 and CNS-0721529.

\section{REFERENCES}

[1] FCC, "Second Report and Order," FCC 08-260, Nov 2008.

[2] IEEE 802.22 Working Group on Wireless Regional Area Networks, http://www.ieee802.org/22/.

[3] A. Ghasemi and E. S. Sousa, "Optimization of Spectrum Sensing for Opportunistic Spectrum Access in Cognitive Radio Networks," in Proc. IEEE CCNC, Jan 2007.

[4] M. Wellens, J. Riihijärvi, M. Gordziel, and P. Mähönen, "Evaluation of Cooperative Spectrum Sensing based on Large Scale Measurements," in Proc. IEEE DySPAN, Oct 2008.

[5] A. W. Min and K. G. Shin, "An Optimal Sensing Framework Based on Spatial RSS-profile in Cognitive Radio Networks," in Proc. IEEE SECON, June 2009.

[6] - "Impact of Mobility on Spectrum Sensing in Cognitive Radio Networks," in Proc. ACM CoRoNet, Sep 2009.

[7] Y.-C. Liang, Y. Zeng, E. C. Peh, and A. T. Hoang, "Sensing-Throughput Tradeoff for Cognitive Radio Networks," IEEE Transactions on Wireless Communications, vol. 7, no. 4, pp. 1326-1337, April 2008.

[8] S. Huang, X. Liu, and Z. Ding, "Optimal Sensing-Transmission Structure for Dynamic Spectrum Access," in Proc. IEEE INFOCOM, April 2009.

[9] R. Chen, J.-M. Park, and J. H. Reed, "Defense against Primary User Emulation Attacks in Cognitive Radio Networks," IEEE Journal on Selected Areas in Communications, vol. 26, no. 1, pp. 25-37, Jan 2008.

[10] A. W. Min, K. G. Shin, and X. Hu, "Attack-Tolerant Distributed Sensing for Dynamic Spectrum Access Networks," in Proc. IEEE ICNP, Oct 2009.

[11] Y. Selén, H. Tullberg, and J. Kronander, "Sensor Selection for Cooperative Spectrum Sensing," in Proc. IEEE DySPAN, Oct 2008.

[12] N. B. Chang and M. Liu, "Optimal Channel Probing and Transmission Scheduling in a Multichannel System," in Proc. ACM MobiCom, Sep 2007, pp. 27-38.
[13] T. Shu and M. Krunz, "Throughput-efficient Sequential Channel Sensing and Probing in Cognitive Radio Networks Under Sensing Errors," in Proc. ACM MobiCom, Sep 2009.

[14] H. Jiang, L. Lai, R. Fan, and H. V. Poor, "Optimal Selection of Channel Sensing Order in Cognitive Radio," IEEE Transactions on Wireless Communications, vol. 8, no. 1, pp. 297-307, Jan 2009.

[15] Q. Zhao, L. Tong, A. Swami, and Y. Chen, "Decentralized Cognitive MAC for Opportunistic Spectrum Access in Ad Hoc Networks: A POMDP Framework," IEEE Journal on Selected Areas in Communications, vol. 25, no. 3, pp. 589-600, April 2007.

[16] J. Jia, Q. Zhang, and X. Shen, "HC-MAC: A Hardware-Constrained Cognitive MAC for Efficient Spectrum Management," IEEE Journal on Selected Areas in Communications, vol. 26, no. 1, pp. 106-117, Jan 2008.

[17] S. Zheng, Y.-C. Liang, P. Y. Kam, and A. T. Hoang, "Cross-layered Design of Spectrum Sensing and MAC for Opportunistic Spectrum Access," in Proc. IEEE WCNC, April 2009.

[18] K. Jain, J. Padhye, V. N. Padmanabhan, and L. Qiu, "Impact of Interference on Multi-hop Wireless Network Performance," in Proc. ACM MobiCom, Sep 2003.

[19] S. Shellhammer, S. Shankar, R. Tandra, and J. Tomcik, "Performance of Power Detector Sensors of DTV Signals in IEEE 802.22 WRANs," in ACM TAPAS, Aug 2006.

[20] H. Su and X. Zhang, "Cross-Layer Based Opportunistic MAC Protocols for QoS Provisionings Over Cognitive Radio Wireless Networks," IEEE Journal on Selected Areas in Communications, vol. 26, no. 1, pp. 118129, Jan 2008.

[21] C. Cordeiro and K. Challapali, "C-MAC: A Cognitive MAC Protocol for Multi-Channel Wireless Networks," in Proc. IEEE DySPAN, April 2007.

[22] IEEE 802.22 WG, "Draft PHY/MAC Specification for IEEE 802.22," IEEE 802.22-06/0069r1, May 2006.

[23] C. Cordeiro, K. Challapali, D. Birru, and S. S. N, "IEEE 802.22: An Introduction to the First Wireless Standard based on Cognitive Radio," IEEE Journal of Communications, vol. 1, no. 1, pp. 38-47, April 2006.

[24] R. Skrekovski, "List Improper Colourings of Planar Graphs," Combinatorics, Probability and Computing, vol. 8, no. 3, pp. 293-299, May 1999.

[25] J. Mo and J. Warland, "Fair End-to-End Window-Based Congestion Control," IEEE Transactions on Networking, vol. 8, no. 5, pp. 556-567, Oct 2000.

[26] E. Arikan, "Some Complexity Results about Packet Radio Networks," IEEE Transactions on Information Theory, vol. 30, no. 4, pp. 681-685, July 1984.

[27] F. Havet and J.-S. Sereni, "Improper Choosability of Graphs and Maximum Average Degree," Journal of Graph Theory, vol. 52, no. 3, pp. 181-199, Feb 2006.

[28] D. Willkomm, S. Machiraju, J. Bolot, and A. Wolisz, "Primary Users in Cellullar Networks: A Large-scale Measurement Study," in Proc. IEEE DySPAN, Oct 2008.

[29] M. Mishra and A. Sahai, "How much white space is there?" EECS Department, University of California, Berkeley, Tech. Rep. UCB/EECS2009-3, Jan 2009.

[30] D. Chen, S. Yin, Q. Zhang, M. Liu, and S. Li, "Mining Spectrum Usage Data: a Large-scale Spectrum Measurement Study," in Proc. ACM MobiCom, Sep 2009.

[31] G. Grimmet and D. Stirzaker, Probability and Random Processes. Oxford University Press, 2nd Edition, 2001.

[32] Part 11: Wireless LAN Medium Access Control (MAC) and Physical Layer (PHY) Specifications: High-speed Physical Layer in the $5 \mathrm{GHz}$ Band, IEEE Std. 802.11a, Sep 1999.

[33] M. Heusse, F. Rousseau, G. Berger-Sabbatel, and A. Duda, "Performance Anomaly of 802.11b," in Proc. IEEE INFOCOM, April 2003.

[34] H. Zheng and C. Peng, "Collaboration and Fairness in Opportunistic Spectrum Access," in Proc. IEEE ICC, May 2005, pp. 3132-3136. 